\title{
Felix Wörner / Ullrich Scheideler / Philip Rupprecht (Hg.), Tonality Since 1950, Stuttgart: Steiner 2017
}

Schlagworte/Keywords: 20. und 21. Jahrhundert; 20th and 21st century; Deutschland und USA; Germany and United States of America; music and technology; Musik und Technologie; Tonalität; tonality

Der zweite Band des auf zwei Bände angelegten Publikationsprojekts zur Tonalität im 20. Jahrhundert ist ebenso wie bereits der erste Band Ergebnis einer internationalen Forschungskooperation zwischen deutschsprachiger und angloamerikanischer Musiktheorie und Musikwissenschaft. ${ }^{1}$ Der im Jahr 2017 erschienene Band Tonality Since 1950 ist aus einer Konferenz hervorgegangen, die 2014 an der Universität Basel stattgefunden hat. Die meisten der darin publizierten Artikel sind Publikationsfassungen von Kongressbeiträgen; einige der Texte sind nachträglich ausschließlich für die Publikation entstanden. ${ }^{2}$

Die Forschung zur tonalen Musik im 20. Jahrhundert hat gegenwärtig Konjunktur: Eine Vielzahl von Publikationen, darunter auch Daniel Harrisons Buch Pieces of Tradition: An Analysis of Contemporary Tonal Music aus dem Jahr 2016, zeigt, dass es eine Tendenz gibt, alternative Narrative zu einer auf die atonale Musik fokussierten Kompositionsgeschichte des 20. Jahrhunderts zu entwickeln. ${ }^{3}$ Da Kompositionsgeschichte des 20. Jahrhunderts stärker als in vorherigen Zeiten sowohl durch Selbstzeugnisse der Komponist*innen als auch durch einen breiten musikpublizistischen Diskurs begleitet wurde, bietet es sich an, ein musikästhetisch so kontroverses Thema wie die Frage nach tonaler Komposition nicht nur analytisch, sondern auch unter Einbezug dieser schriftlichen Quellen aufzuarbeiten. ${ }^{4}$ So sind es, während im ersten Band einige Beiträge explizit

1 Vgl. meine Rezension des ersten Bands in der ZGMTH (Sprick 2015).

2 Dass beide Bände zusammenhängen, zeigt sich nicht zuletzt daran, dass dem zweiten Band ein gemeinsames Register für beide Bände beigegeben ist.

3 Harrison 2016.

4 Vgl. dazu Sprick 2014. musiktheoretischen Schriften gewidmet sind, im zweiten Band in erster Linie Selbstzeugnisse von Komponist*innen und musikästhetische Diskurse, die die analytischen Überlegungen kontextualisieren. Diese Selbstzeugnisse umfassen ein breites Spektrum von Textgattungen: Programmhefttexte, Rundfunkmanuskripte, Interviews, ästhetische Grundsatzerklärungen, Darstellungen eigener kompositorischer Prinzipien, Analysen von Werken anderer Komponist*innen und sogar wissenschaftliche Abhandlungen. Insbesondere für die zweite Hälfte des 20. Jahrhunderts und das beginnende 21. Jahrhundert hat diese Tendenz noch einmal zugenommen, was man an den vielfältigen Selbstzeugnissen sehen kann, die mittlerweile nicht mehr an unterschiedlichen Orten zerstreut sind, sondern zum großen Teil in edierten Sammelbänden vorliegen.

Vor diesem Hintergrund erweist sich die Entscheidung der Herausgeber als sehr überzeugend, in dem Band - wie bereits in dem ersten der beiden Bände - Autor*innen zu versammeln, die zu etwa gleichen Anteilen die Disziplinen Musiktheorie und Musikwissenschaft repräsentieren. Das Ziel der Beiträge ist letztendlich immer die analytische Auseinandersetzung, doch weniger im Hinblick auf einen Anspruch, eine allgemeine Methodologie für die Analyse tonaler Musik im 20. Jahrhundert zu entwickeln - wie es Harrison in seinem Buch versucht ${ }^{5}$-, als vielmehr im Hinblick darauf, der individuellen Situation einzelner Komponist*innen gerecht zu werden. Oder in anderen Worten ausgedrückt: Anders als Harrison, der trotz der Heterogenität tonalen Komponierens im 20. Jahrhundert zu einer musikalischen Theoriebildung beitragen möchte, was Pieces of Tradition zu einem dezidiert

5 Vgl. dazu auch die Rezension von Ullrich Scheideler in der ZGMTH (Scheideler 2018). 
musiktheoretischen Buch macht, repräsentiert Tonality Since 1950 vielmehr die Schnittstelle beider Disziplinen.

Die Herausgeber verfolgen das Ziel, die sehr heterogenen Ausprägungen tonaler Musik in der Musik seit 1950 in ihrer Individualität und unter einer sehr genauen Einbeziehung und Darstellung ihres jeweiligen historischen Ortes zu behandeln. Diesen Ansatz haben die Herausgeber bereits im ersten Band verfolgt, allerdings mit einem anderen Referenzpunkt. Knapp zusammengefasst kann man das Anliegen des ersten Bands als Versuch beschreiben, die Erzählung vom `Ende` der Tonalität um 1910 zu relativieren und ein alternatives Narrativ vorzustellen, das den Blick auf die Kontinuität tonalen Komponierens bei Komponisten wie Jean Sibelius, Claude Debussy, Aaron Copland, Sergej Prokof'ev, Francis Poulenc und Michael Tippett lenkt. Der zweite Schwerpunkt des Bandes war die Reflexion von Tonalität in dezidiert musiktheoretischen Schriften, in denen Tonalität - unabhängig von den Entwicklungen im zeitgenössischen Komponieren - eine Konjunktur erlebt, die sich insbesondere in den Schriften von Arnold Schönberg, Heinrich Schenker, Ernst Kurth und Hugo Riemann widerspiegelt. Dabei ist es kein Zufall, dass für die erste Jahrhunderthälfte der musiktheoretische Diskurs eine entscheidende Rolle spielt, was sich in der Präsenz der eben erwähnten Theoretiker niederschlägt. Aufgrund der Ausdifferenzierung des theoretischen Diskurses lassen sich für die zweite Jahrhunderthälfte nicht mehr einzelne Theoretiker*innen benennen, in deren Denken sich entscheidende Aspekte einer Theorie von Tonalität widerspiegeln, sodass es nicht verwundert, dass die Darstellung sich auf die individuelle Diskussion einzelner Ansätze verlagert.

Ähnlich wie im ersten Band stellen die Herausgeber den thematisch sehr diversen Beiträgen eine Einleitung voran, in der sie einige Reflexionen zum Phänomen der Tonalität für die zweite Hälfte des 20. Jahrhunderts liefern. Im Unterschied zum ersten Band und der darin gegebenen Definition, derzufolge unter Tonalität ganz allgemein »the awareness of key in music " ${ }^{6}$ verstanden wird, erscheint das Tonalitätsverständnis im zweiten Band dahingehend erweitert, dass auch musikalische Zusammenhänge als stonal bezeichnet werden, die beispielsweise auf Tonfeldern beruhen. Darüber hinaus wird der Untersuchungsgegenstand ausdrücklich auf den Bereich der Pop- und Rockmusik erweitert. Geographisch bezieht sich der Band auf Europa, die USA und die Sowjetunion. Wie schon im ersten Band geht es den Herausgebern in erster Linie darum, "continuities with past practices « zu betonen, »rather than sudden breaks" (14). Und auch die Aussage, dass der Zeitraum das Datum im Titel eine Periode von »multiple ongoing engagements with tonality over the past half-century" markiert und nicht "the aftermath of some singular collapse« (ebd.), zeigt das allgemeine Anliegen, mit dem Band zu einer breiten und vielschichtigen Darstellung der Kompositionsgeschichte des 20. Jahrhunderts beizutragen. Komponisten wie György Ligeti, Gérard Grisey, Alfred Schnittke und Hans Werner Henze werden mit Äußerungen zitiert, die das Gefühl eines Verlusts, das mit dem vermeintlichen Ende der Tonalität einhergeht, illustrieren. Vor diesem Hintergrund ist es nur allzu verständlich, dass die Herausgeber mit dem Band nicht das Ziel verfolgen, eine "comprehensive shistory< of tonality in the post-1950 period « (ebd.) zu liefern. Die Absicht ist vielmehr, eine Grundlage für weitere Forschungen in Form einer Reihe von Fallstudien zu legen.

Die Abfolge der Einzelbeiträge gliedert sich in drei Teile. Unter der Überschrift »Concepts and Contexts « sind im ersten Teil vier Artikel versammelt, die stärker als die später folgenden Fallstudien übergreifende Aspekte thematisieren. Die Tatsache, dass dabei zwei Texte der ,E-Musikı gewidmet sind und zwei Texte der 'Popularmusikı, zeigt die eben beschriebene Verschiebung des Fokus in der zweiten Hälfte des 20. Jahrhunderts. Sowohl Ulrich Mosch (»Foundations or Mere Quotation? Conditions for Applying the Tonality Concept to Music After 1950«; 27-49) als auch Wolfgang Rathert (»Total Tonality or Tonal Totality: A Compositional Issue in Music After 1945«; 51-63) schlagen einen jeweils unterschiedlich erweiterten Begriff von Tonalität vor bzw. denken über Bedingungen für eine solche Begriffserweiterung nach.

Während Ulrich Mosch in erster Linie versucht, eine griffige Definition von Tonalität zu 
liefern, und diese mit Musikbeispielen aus dem 20. Jahrhundert abgleicht, diskutiert Wolfgang Rathert in seinem Beitrag Stationen des Tonalitätsdiskurses in der zweiten Hälfte des 20. Jahrhunderts. In einem ersten - und längsten Abschnitt stellt er Paul Hindemiths aus der Perspektive der Avantgarde »reaktionäre« Position derjenigen Schönbergs gegenüber und rekurriert hier insbesondere auf Hindemiths "sound-related [...] conception of tonality" (59). Damit bringt Ratherts Text Hindemith als eine zentrale Stimme im Tonalitätsdiskurs der 1950er Jahre wieder ins Bewusstsein, insbesondere seine Idee der "tonal totality". Als weitere Beispiele in Ratherts Text fungieren neben Pierre Boulez auch Luigi Nono, Helmut Lachenmann und György Ligeti. Interessant an dieser Auswahl ist, dass alle diese Komponisten in der Regel nicht mit tonalen Tendenzen identifiziert werden. Ein letzter Exponent von Hindemiths »tonal totality « ist in den Augen Ratherts Leonard Bernstein, der in den 1940er Jahren sowohl von einer intensiven HindemithRezeption geprägt, zeitgleich aber auch intensiv vom Jazz beeinflusst war und überdies Schönbergs Zwölftonmusik analysiert hat. In seinem Fazit betont Rathert, dass sich die schwierige Situation nach 1950 exemplarisch für die Einleitung in die Komplexität der Fragestellungen eignet, die in dem Band thematisiert werden.

Joseph Auners Text »The Stopped Clock: Tape Loops, Synthesizers, and the Transfiguration of Harmony« (65-87) kann als Fortführung von Ideen aus seinem Beitrag im ersten Band verstanden werden. Durch den Einbezug der Ebene der Technologie beschäftigt sich Auner mit einem sehr interessanten und für den deutschsprachigen Kontext ungewöhnlichen Repertoire. Eine zentrale Frage für Auner ist, wie technische Möglichkeiten den Umgang mit Tonalität verändern. Die wesentlichen technischen Errungenschaften und deren Bedeutung für den Umgang mit Tonalität sind der tape loop und der modular synthesizer. Auner verweist insbesondere auf statische Zustände, die durch Loops und Ostinati hervorgerufen werden können. Mit der Diskussion von Beispielen von Grisey und Giacinto Scelsi in diesem Zusammenhang betont Auner die enge Verbindung bestimmter Entwicklungen in der neuen
Musik mit progressiven Entwicklungen in der Pop-Musik.

Fokussierter geht Nicole Biamonte in ihrem Text »Pop/Rock Tonalities« (89-101) vor. Der teilweise grundlegende Duktus des Textes zeigt, dass die Autorin der Auffassung ist, die Diskussion von Aspekten der Pop/Rock-Musik brauche eine stärkere Einführung als die anderer Musikstile. Während auch Biamonte erwähnt, dass viele Forscher*innen betont haben, Pop/Rock-Musik müsse nach ihren ganz eigenen Bedingungen analysiert werden, liefert sie selbst in ihrem Text ein Beispiel dafür, dass man für die Analyse von Popularmusik - insbesondere die Analyse der Harmonik - ähnliche Verfahren anwenden kann wie bei sklassischer Musik. Die Frage der analytischen Methodologie muss hier also sehr eindeutig von ihrer jeweiligen Fragestellung her beantwortet werden. Pop/Rock-Musik hat in der Regel ein tonales Zentrum, sodass "structural notes are normally part of the harmony or are emphasized in the melody« (90). Es fällt auf, dass Biamonte keinerlei deutschsprachige Literatur rezipiert, eine Feststellung die hier nicht unbedingt als Vorwurf gemeint ist, sondern eher den gegenwärtigen Zustand in der Pop/Rock-Forschung illustriert.

Insgesamt repräsentieren diese vier Artikel des einleitenden Abschnitts in exemplarischer Weise die gegenwärtigen Zustände der jeweiligen Disziplinen in den einzelnen Ländern (deutschsprachige Musikwissenschaft, US-amerikanische Musicology und Music Theory). Hier wäre die Einbeziehung einer Position aus der deutschsprachigen Musiktheorie wünschenswert gewesen.

Der zweite Teil des Bands versammelt vier Texte über Komponisten, die in den 1950er und 1960er Jahren in den USA und Deutschland Popularität erlangt haben. Die Beiträge über Hanns Eisler, Hans Werner Henze, George Rochberg und Morton Feldman repräsentieren vier sehr unterschiedliche Komponisten und Ästhetiken. Jeder Text ist für sich genommen sehr interessant und analytisch fruchtbar, allerdings stellt sich nach der Lektüre die Frage, was die Auswahl dieser vier Komponisten über die generelle Situation der tonalen Komposition in der Jahrhundertmitte aussagt. Auch hier ist es wieder so, dass alle vier Komponisten im Hinblick auf die sie begleitenden Diskurse kontex- 
tualisiert werden. Ein wiederkehrendes Moment ist also die offensichtliche Notwendigkeit, kompositorische Entscheidungen vor dem Hintergrund der herrschenden Avantgarde-Ästhetik zu verstehen.

Unabhängig von dieser generellen Tendenz nähern sich die vier Autor*innen ihren Gegenständen auf sehr unterschiedliche Weise. Während etwa Thomas Ahrend in seinem Beitrag ") Das Wunderland: Tonality and (Political) Topography in Eisler's Songs Around 1950 « (105-127) einen Schwerpunkt auf das Verständnis der rahmenden Diskurse legt, steht in Ullrich Scheidelers Beitrag "Tonality in Henze's Music of the 1950s and Early 1960s" (129-152) die analytische Perspektive im Vordergrund. Auch Scheideler beginnt einleitend mit der Darstellung von Henzes komplexer Position im deutschen Musikleben der 1950er Jahre, ohne die Henzes Musik nicht adäquat verständlich ist.

Wie bereits der Titel des dritten Teils andeutet (»Processes, Objects, Functions, and Resonances«), geht es hier weniger um eine inhaltliche Fokussierung als um eine möglichst breite und diverse Aufarbeitung des Phänomens der Tonalität seit 1970. Thematisiert werden mit Steve Reich (Keith Potter), Oliver Knussen (Philip Rupprecht), Alfred Schnittke (Peter J. Schmelz), Kaija Saariaho (Eric Drott), Georg Friedrich Haas (Simone Heilgendorff), Thomas Adès (Felix Wörner) und György Kurtág (Volker Helbing) in erster Linie Komponist*innen, die auch im gegenwärtigen Musikleben eine wichtige Rolle spielen. Ohne diese Auswahl zu kritisieren, wäre dennoch anzumerken, dass hier auffälligerweise keine deutschen Komponist*innen diskutiert werden, die dem Umfeld der "Neuen Einfachheit» und damit einer wesentlichen Strömung der Musik der zweiten Hälfte des 20. Jahrhunderts in Deutschland entstammt.

Wie schon beim ersten Band wird auch bei seiner Fortsetzung die selbstverständlich unerfüllbare Erwartung enttäuscht, in dem Band einen enzyklopädischen Überblick über Tonalität in der zweiten Hälfte des 20. Jahrhunderts zu bekommen. Auch der zweite Band versammelt eine Reihe sehr interessanter Fallstudien, die sich hinsichtlich des untersuchten Repertoires abseits der teilweise bereits ausgetretenen Pfade der Analyse neuer Musik aus dem
Umfeld der Darmstädter Schule und anderer Avantgarde-Strömungen bewegen. Dabei ist in dem Band eine Grundtendenz spürbar, die sich bereits in der Einleitung angedeutet hat: die Intention, jenen Komponist*innen endlich zu ihrem Recht zu verhelfen, deren Position im analytischen und ästhetischen Musikdiskurs bisher unterbelichtet ist. Dieses Anliegen ist mit Sicherheit in vielen Fällen auch berechtigt. Allerdings hätte man sich gelegentlich gewünscht, Verständnis auch für jene Positionen aufzubringen, für die das Schreiben tonaler Musik nach dem Zweiten Weltkrieg keine Option mehr gewesen ist. In diesem Sinne wäre beispielsweise ein Artikel willkommen gewesen, der eine provokative Gegenposition formuliert hätte.

Auf der Ebene der Darstellung hätte eine etwas stärkere Angleichung der Beiträge, beispielsweise im Hinblick auf die Länge der Fußnoten und das Verhältnis von Kontextualisierung und Analyse, zu einem einheitlicheren Bild geführt. Auf der anderen Seite reflektieren Differenzen unter diesem Aspekt auch die sehr positiv zu bewertende Tatsache, dass sich in diesem Band - wie bereits im ersten Teil - die Disziplinen Musiktheorie und Musikwissenschaft auf Augenhöhe begegnen, insofern die eben erwähnten Unterschiede in erster Linie auf unterschiedliche Traditionen und Fragestellungen in den beiden Fächern zurückzuführen sind. Dennoch hätte, unabhängig von den Unterschieden in der Darstellung, ein Rückbezug auf eine allen Texten gemeinsame Leitfrage oder eine Ausgangsthese vor dem Hintergrund der großen Heterogenität der thematisierten Musik produktiv sein können.

Wie bereits der Band über die erste Hälfte des 20. Jahrhunderts bietet auch der ebenfalls sehr sorgfältig lektorierte und gestaltete Fortsetzungsband eine beeindruckende Vielfalt an Perspektiven auf den Gegenstand. Fast noch stärker als in der ersten Hälfte des 20. Jahrhunderts wird deutlich, dass Tonalität bzw. tonale Elemente bei vielen Komponist*innen nach 1950 eine Möglichkeit unter vielen sind, ihre ästhetischen Ideen umzusetzen. Die kontinuierliche Einbindung in musikästhetische Diskurse zeigt allerdings, dass das Thema auch in der zweiten Hälfte des 20. Jahrhunderts immer noch Potenzial für Kontroversen hat und es jeweils notwendig zu 
sein scheint, die Entscheidung für die Verwendung tonaler Elemente zu begründen bzw. deren Verwendung im Diskurs über diese Werke zu erwähnen. Beide Bände zusammen können bereits jetzt als eine Referenzpublikation für ein Thema bezeichnet werden, das in der musikwissenschaftlichen und musiktheoretischen Literatur zum 20. Jahrhundert bislang zu wenig Raum bekommen hat. Gemeinsam mit den zu Beginn der Rezension erwähnten Publikationen zur Tonalität im 20. Jahrhundert kann die Doppelpublikation den Ausgangspunkt für weitere Forschungen auf diesem Gebiet darstellen.

Jan Philipp Sprick

\section{Literatur}

Harrison, Daniel (2016), Pieces of Tradition. An Analysis of Contemporary Tonal Music, New York: Oxford University Press.

Scheideler, Ullrich (2018), »Daniel Harrison, Pieces of Tradition. An Analysis of Contemporary Tonal Music, New York: Oxford University Press 2016", ZGMTH 15/2, 243-251. https://doi.org/10.31751/995 (15.12.2019)

Sprick, Jan Philipp (2014), »Der Komponist als Autor. Überlegungen zur Didaktik der Analyse Neuer Musik«, in: Kreativitätsportal Musik, Universität der Künste Berlin. https://www.udk-berlin.de/fileadmin/2_deze ntral/FR_Musiktheorie/kPortal/kportal_sprick _komponistalsautor.pdf (15.12.2019)

Sprick, Jan Philipp (2015), »Felix Wörner / Ullrich Scheideler / Philip Rupprecht (Hg.), Tonality 1900-1950. Concept and Practice, Stuttgart: Steiner 2012«, ZGMTH 12/1, 147-150. https://doi.org/10.31751/838 (15.12.2019)

Wörner, Felix / Ullrich Scheideler / Philip Rupprecht (Hg.) (2012), Tonality 19001950. Concept and Practice, Stuttgart: Steiner.

Sprick, Jan Philipp (2019): Felix Wörner / Ullrich Scheideler / Philip Rupprecht (Hg.), Tonality Since 1950, Stuttgart: Steiner 2017. ZGMTH 16/2, 193-197.

https://doi.org/10.31751/1025

(C) 2019 Jan Philipp Sprick (jan.sprick@gmx.net)

Hochschule für Musik und Theater Hamburg

Dieser Text erscheint im Open Access und ist lizenziert unter einer Creative Commons Namensnennung 4.0 International Lizenz.

This is an open access article licensed under a

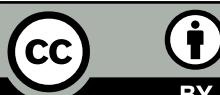

Creative Commons Attribution 4.0 International License.

eingereicht / submitted: 09/11/2019

angenommen / accepted: 12/11/2019

veröffentlicht / first published: 23/12/2019

zuletzt geändert / last updated: 10/01/2020 